\title{
Automatic identification of species with neural networks
}

A new automatic identification system using photographic images has been designed to recognize fish, plant, and butterfly species from Europe and South America. The automatic classification system integrates multiple image processing tools to extract the geometry, morphology, and texture of the images. Artificial neural networks (ANNs) were used as the pattern recognition method. We tested a data set that included 740 species and 11,198 individuals. Our results show that the system performed with high accuracy, reaching $91.65 \%$ of true positive fish identifications, $92.87 \%$ of plants and $93.25 \%$ of butterflies. Our results highlight how the neural networks are complementary to species identification, which is useful in today's taxonomic crisis. 


\section{Automatic identification of species with neural networks.}

2 Andrés Hernández-Serna ${ }^{a, b}$ * and Luz Fernanda Jiménez-Segura ${ }^{a}$

3 a Grupo de Ictiología; Instituto de Biología, Universidad de Antioquia, Medellín, Colombia

$4{ }^{b}$ Department of Biology, University of Puerto Rico-Río Piedras, San Juan, Puerto Rico.

5 *Corresponding autor: Andres Hernández-Serna, PO Box 23360, Department of Biology,

6 University of Puerto Rico, San Juan, PR 00931-3360, USA

7 e-mail: andres137@gmail.com

\section{ABSTRACT}

9 A new automatic identification system using photographic images has been designed to recognize

10 fish, plant, and butterfly species from Europe and South America. The automatic classification

11 system integrates multiple image processing tools to extract the geometry, morphology, and

12 texture of the images. Artificial neural networks (ANNs) were used as the pattern recognition

13 method. We tested a data set that included 740 species and 11,198 individuals. Our results show

14 that the system performed with high accuracy, reaching $91.65 \%$ of true positive fish

15 identifications, $92.87 \%$ of plants and $93.25 \%$ of butterflies. Our results highlight how the neural

16 networks are complementary to species identification, which is useful in today's taxonomic

17 crisis.

18 Keywords: Fish, plant, butterflies, neural network, feature extraction, digital image, and species

\section{INTRODUCTION}


20 Currently, species identification is a taxonomic challenge and an integral process of all biological

21 research, which generates important information for biodiversity conservation. Difficulty

22 identifying species and ambiguity in the species concept, are seriously affecting our ability to

23 estimate levels of biodiversity (Gaston \& O'Neill, 2004). The Global Taxonomy Initiative

24 highlights the knowledge gaps in our taxonomic system due to the shortage of trained

25 taxonomists and curators; these deficiencies reduce our ability to understand, use, and conserve

26 biological diversity. High levels of global biodiversity and a limited number of taxonomists

27 represents significant challenges to the future of biological study and conservation. The main

28 problem is that almost all taxonomic information exists in languages and formats not easily

29 understood or shared without a high level of specialized knowledge and vocabularies. Thus,

30 taxonomic knowledge is localized within limited geographical areas and among a limited number

31 of taxonomists. This lack of accessibility of taxonomic knowledge to the general public has been

32 termed the "taxonomic crisis" (Dayrat, 2005).

33 Recently, taxonomists have been searching for more efficient methods to meet species

34 identification requirements, such as developing digital image processing and pattern recognition

35 techniques. These methods automatically identify species based on extracting unique image

36 shape information that distinguishes them by taxonomic groups. Researchers currently have

37 recognition techniques for insects, plants, spiders, and plankton (Gaston \& O'Neill, 2004). This

38 approach can be extended even further to field-based identification of organisms such as fish

39 (Strachan, Nesvadba \& Allen, 1990; Storbeck \& Daan, 2001; White, Svellingen \& Strachan,

40 2006; Zion, Alchanatis, Ostrovsky, Barki \& Karplus, 2007; Hu, Li, Duan, Han, Chen \& Si, 2012),

41 insects (Mayo \& Watson, 2007; O'Neill, 2007 ; Kang, Song \& Lee, 2012), zooplankton

42 (Grosjean, Picheral, Warembourg \& Gorsky, 2004) and plants (Novotny \& Suk, 2013). These

43 methods are helpful in alleviating the "taxonomy crisis". In this research, we present a new 
44 methodology for the identification of different taxonomic groups to the species level for fish, 45 plants, and butterflies.

46 We designed a simple and effective algorithm (preprocess solution) and defined a range of new

47 features that use pattern recognition with artificial neural network designs (ANN). Our

48 experiments are outlined, discussed, and important conclusions on automatic species image

49 identification are summarized.

50 MATERIALS AND METHODS

\section{Images}

52 Image data in this study was taking from two sources: natural history museum records, and online

53 databases. Analyses from each collection were done with respect to country. Ichthyology

54 collections from Colombia were compiled from the Instituto de Investigaciones Marinas y

55 Costeras (INVEMAR), the Colección de Referencia Biología Marina Universidad del Valle

56 (CRBMUV), and the Coleccion Ictiologica Universidad de Antioquia (CIUA). Ichthyology

57 collections from Brazil were found in the Museu de Zoologia da USP (MZUSP), the Instituto

58 Nacional de Pesquisas da Amazônia Manaus (INPA), and the Museu Nacional Rio de Janeiro

59 (MNRJ). Image data from Spain came from the Museo Nacional de Ciencias Naturales Madrid

60 (MNCN). We tested a data set that included a total of 740 species and 11,198 individuals of fish,

61 plants, and butterflies. Fish specimen images were taken using a Cannon EOS 6dD one-use

62 camera with a $1280 \times 960$ pixel resolution. 697 total fish species, previously identified by

63 experts, were photographed (see Fig. 1 for a subset of photographed species). Images of 32 plant

64 species were downloaded from the Flavia database (http://flavia.sourceforge.net/) (see Fig.2).

65 Image data for 11 species of butterflies were downloaded from the MorphBank database

66 (http://www.morphbank.net/) (see Fig. 3.). 


\section{System development}

68 Based on pattern recognition theory (Marqués de Sá, 2001) and basic computer-processing

69 pathways used in typical automated species identification systems (Gaston \& O'Neill, 2004), we 70 designed a system for automatic individual identification at the species level (Fig. 4). In a novel

71 way, our system shares preprocess and extraction components with both the training and

72 recognition processes. Features of training images are used to build a model of the classification

73 progress pattern after feature extraction. These features and the trained model are then recorded

74 in the database and incorporated in the analysis of subsequent photos. This process uses two

75 types of data to model features of recognition files and results in better species identification

76 results. The following sections provide implementation details for each step in Fig. 4. Due to its

77 size, a list of features could not be included in this manuscript, but is alternatively available upon 78 request.

\section{Image preprocessing}

80 Image heterogeneity in terms of orientation, size, brightness, and illumination was common (Fig.

81 5.1). Image background was removed with Grabcut's algorithm (Rother, Kolmogorov \& Blake,

82 2004) (Fig. 5.2) and converted to grayscale (Fig. 5.3). Different filters were applied to improve

83 the image by removing image noise; the filters used were smooth and median (Fig. 5.4 and 5.5),

84 and the image was then reduced to one of two possible levels, 0 or 1 (Fig. 5.6). Next, the

85 processed image was brought to a contour (Fig. 5.7) and then a skeleton (Fig. 5.8). All of these

86 processes were performed for each taxonomic group using the image processing in MATLAB

87 R2009b.

\section{Feature extraction}


89 Feature extraction greatly influences species identification from image processing. Features

90 should represent taxonomic information and be easily acquired from data images. A series of

91 geometrical, morphological, and texture features, unique to species, are used in our automatic

92 identification system; these features can be efficiently extracted with image processing. Fifteen

93 intuitive features were used in the system and are described below:

\section{Geometrical}

95 Geometric features contain information about form, position, size, and orientation of the region.

96 The following are some geometric features that are commonly used in pattern recognition.

97 1- Area is the total number of pixels of the study area, and is defined as:

$$
A(s)=\int_{x} \int_{y} I(x, y) d y d x
$$

98 I (x, y) depends on the limits of the shape (see figure 5.7).

99 2-Perimeter. The number of pixels that belong to the edge of the region (see figure 5.8). In other

100 words, it is the curve that encloses a region S, defined as:

$$
P(s)=\int_{t} \sqrt{x^{2}(t)+y^{2}(t)} d t
$$

101 3-Diameter. Value representing the diameter of a circle with the same area as the region.

102 4-Compatibility. The efficiency of the contour or perimeter $\mathrm{P}(\mathrm{s})$ that encloses an area $\mathrm{A}(\mathrm{s})$

$$
C(s)=\frac{4 \pi A(s)}{P^{2}(s)}
$$

103 5- Compactness. The efficiency with which area $\mathrm{A}(\mathrm{s})$ encloses an object is determined by $\mathrm{P}(\mathrm{s})$ 


$$
C o(s)=\frac{P^{2}(s)}{4 \pi A(s)}
$$

104 6- Solidity. The scalar specifying the proportion of the pixels in the convex hull that are also in 105 the region. This property is supported only for 2-D input label matrices.

106 Solidity. The number of pixels, specified in terms of area/scalar.

\section{Texture}

108 Textures are important visual patterns for homogeneous description of regions. Intuitive measures

109 provide properties such as smoothing, roughness, and regularity (Glasbey, 1996). Textures

110 depend on the resolution of the image and can follow two approaches: statistical and frequency.

111 We use the statistical approximation in which statistical values are analyzed first order (on the

112 histogram) and second order (on the co-occurrence matrix).

113 Statistical first order is obtained from the gray level histogram of the image. Each value is

114 divided by the total number of pixels (area) and has a new histogram representing the probability

115 that a determined gray level is displayed in the region of interest.

116 Obtained properties:

117 7-Median

$$
\mu=\sum_{x=1}^{n} x h(x)
$$

118 8-Variance

$$
\delta^{2}=\sum_{x=1}^{n}\left(x-\mu^{2}\right) h(x)
$$


119 Statistical second order is the matrix spatial dependence of gray levels or co-occurrence matrices.

120 Given a vector of polar coordinates, $\delta=(r, \theta)$, one can calculate the conditional probability

121 that two properties appear separated by a given distance $\delta, P_{\delta}$ using an angle $\theta$ of -45 and a

122 distance $r$ equal to one pixel. The features that are extracted from this matrix are:

123 9- Uniformity

$$
\sum_{x=1}^{n} \sum_{y=1}^{n} P_{\delta}(x, y)^{2}
$$

124 10- Entropy co-occurrence

$-\sum_{x=1}^{n} \sum_{y=1}^{n} P_{\delta}(x, y) \log P_{\delta}(x, y)$

125 11- Homogeneity

$$
\frac{\sum_{x=1}^{n} \sum_{y=1}^{n} P_{\delta}(x, y)}{1+|x-y|}
$$

126 12- Inertia

$$
\sum_{x=1}^{n} \sum_{y=1}^{n} P_{\delta}(x, y)(x-y)^{2}
$$

\section{Morphological}


128 The morphological features are those that concentrate on the organization of pixels. They perform

129 a comprehensive description of the region of interest. They fall into two categories: two-

130 dimensional Cartesian moments and normalized central moments.

131 Two-dimensional Cartesian moments are variable at minor order, and initiate at zero at higher

132 orders. The moment of order $\mathrm{p}$ and $\mathrm{q}$ of a function $\mathrm{I}(\mathrm{x}, \mathrm{y})$ is defined as:

$$
m_{p q}=\int_{-\infty}^{\infty} \int_{-\infty}^{\infty} x^{p} y^{q} I(x, y) d x d y
$$

133 The parameters $p$ and $q$ denote the order of the moment. When $p=0$ and $q=0$, which determines

134 the center of mass or gravity of the overall function in binary images, the center of mass or

135 gravity of the region under study is:

$\bar{x}=\frac{m_{10}}{m_{00}} \quad \bar{y}=\frac{m_{01}}{m_{00}}$

136 The center of mass or gravity can define the central moments that are invariant to displacement or

137 translation of the image's region of interest defined as:

$u_{p q}=\sum_{x} \sum_{y}(x-\bar{x})^{p}(y-\bar{y})^{q} I(x, y) \Delta A$

138 Where $\Delta A$ is the area of a pixel.

139 Normalized central moments are invariant to scale which is defined as:

$$
n_{p q}=\frac{u_{p q}}{u^{\gamma} 00}
$$

140 Where $\gamma=\frac{p+q}{2} \forall p+q \geq 2$ 
141 The above equations can be defined by seven moments that are invariant to rotation, translation,

142 and scale changes, known as the Hu invariant set of moments (Hu, 1962). In this study, we used

143 the first Hu moment defined as:

$144 \quad 13-H u 1$

$\varphi_{1}=m_{20}+m_{02}$

145 Normalized central moments can be generated by related moment invariants "AMI" (Flusser \&

146 Suk, 1993), based on the theory of algebraic invariants and invariants under general affine

147 transformation. We used two of the four invariants associated with discriminant character

148 moments defined as:

149 14-Amil

$I_{1}=\frac{u_{20} u_{02}-u_{11}^{2}}{u_{00}^{4}}$

$150 \quad 15-A m i 2$

$I_{2}=\frac{u_{30}^{2} u_{03}^{2}-6 u_{30} u_{21} u_{12}+4 u_{30} u_{12}^{3}+4 u_{21}^{3} u_{03}-3 u_{21}^{2} u_{12}^{2}}{u_{00}^{10}}$

151 These moments enable a high degree of insensitivity to noise that is not altered by rotation,

152 translation, or staggering.

153 The use of the above 15 features (Table 1) has two advantages. First, the features can express the

154 structure of the individual's body, which is important for the identification at species level.

155 Second, our features were elaborately chosen to avoid using feature optimization methods like

156 adapted fuzzy reasoning (Lancieri \& Boubchir, 2007). We designed and realized automatic 
157 extraction algorithms to compute the values of these features so that all variables and features

158 could be calculated automatically.

\section{Neural Network}

160 A neural network is defined as a parallel computer model composed of a large number of

161 adaptive processing (neural) units which communicate via interconnections with variables. A

162 multiple layer network has one or more layers (neurons) that enable the learning of complex

163 tasks by progressively extracting more meaningful features from the input image patterns ( $\mathrm{Wu}$,

164 1997). Compared to other machine learning methods, neural networks learn slower but predict

165 faster and have very good models presenting nonlinear data. The simple perceptron is assigned

166 multiple inputs but generates a single output, similar to different linear combinations that depend

167 on input weights and generate a linear activation function (Rosenblatt, 1958 ). Mathematically,

168 the neural network can be described with the following equation:

$$
y=\varphi\left(\sum_{i=1}^{n} w_{i} * x_{i}+b\right)
$$

$169 \boldsymbol{W}_{i}$ : weight vector, $\boldsymbol{X}_{i}$ : input vector, $\boldsymbol{b}:$ bias activation function.

170 A multilayer perceptron consists of a set of source nodes containing one or more input layer and a

171 set of hidden-node outputs. The input signal propagates through the network layer by layer

172 (Zhang, Patuwo \& Hu, 1998). Fig. 6 presents a diagram of the multilayer neural network.

173 The neural network structure is composed of $\mathrm{N}$ inputs $\mathrm{N}=\left[\mathrm{N}_{1}, \mathrm{~N}_{2}, \ldots, \mathrm{N}_{\mathrm{n}}\right]$, a hidden layer $\mathrm{h}$ and

174 an output vector $S=\left[\mathrm{S}_{1}, \mathrm{~S}_{2}, \ldots, \mathrm{S}_{m}\right]$. Each $\mathrm{S}_{\mathrm{i}}$ is assessed by a single step that transforms the

175 vector $\mathrm{S}$ binary signal $[0,1]$. A supervised training phase, or sigmoid activation, is based on the

176 back propagation algorithm in which the weights and biases are updated in the direction of the 
177 negative gradient of the performance and then updated in the opposite direction( Werbos, 1974;

178 Rumelhart, Hinton \& Williams, 1986; Parker, 1987; Smith \& Brier, 1996;). The sigmoid

179 activation function for the hidden layer and output layer is determined by the following equation:

$$
f(x)=\frac{1}{1+e^{-x}}
$$

180 In this study, the number of input neurons is determined by the number of descriptors that are

181 available in each pattern, which in this case is $\mathrm{N}=15$ (see variables section). The number of

182 neurons in the hidden layer, $h$, has been experimentally determined from the error set data

183 searching for the general training date of the ANN. The number of output neurons is determined

184 by the number of species classified in each database.

185 All features were extracted from images and defined according to the above mentioned methods.

186 We tested different species from various taxonomic groups, using the developed neural network

187 systems. The results of the main tests with different test species are listed below in results.

\section{RESULTS}

189 Experiments were divided into two groups: 1) images from the training group were used for

190 building the classifications of the model; 2) images from the test group were used for the

191 reorganization and testing of the developed model.

192 To determine the optimal number of neurons given a data image, the relationship between the

193 identification success rate and the number of neurons was explored. Fig. 7 shows this relationship

194 for the different configurations considered. We finally established our networks with 200 neurons

195 for FC-MZUSP, 180 neurons for FC-INPA, 60 neurons for FC-MNRJ, 250 neurons for FC-

196 INVEMAR, 60 neurons for FC-CIUA, 300 neurons for FC-CRBMUV, 250 neurons for FC- 
197 MNCN, 60 neurons for FLAVIA, and 35 neurons for BUTTERFLIES (see Table 2). The number

198 of generations (i.e. a finite set of input patterns presented sequentially) for training and testing the

199 ANNs was variable in the different collections between 50,000 to 140,000. It is evident the High

200 number of neurons and generations to process the information of the images in each collection.

201 However, the speed of modern PCs allows easy application of the employed architectures.

202 Table 3 shows the performance average of the artificial neural networks using image data and the 20315 analyzing features. The data set was randomly divided into $60-70-80-90 \%$ training images, 204 resulting in 40-30-20-10\% test images. The results with the highest average accuracy for species 205 identification were networks using $80-90 \%$ training and $20-10 \%$ test images. For these tests, the 206 declared success rate was related to the number of species. Recognition became more difficult 207 with increased species number, as observed in the fish result collections from MZUSP, INPA, 208 INVEMAR, CRBMUV, and MNCN which averaged below 90\% recognition.

\section{DISCUSSION}

210 Similar to previous findings (Strachan et al., 1990; Storbeck \& Daan, 2001; White et al., 2006;

211 Zion et al., 2007; Novotny \& Suk, 2013), the neural network used classified species from image

212 data. However, most other studies only employ databases with low levels of species richness

213 usually spanning many different orders and families and are easily classified due to distinct

214 differences in morphological characteristics. Our neural network builds on the work of these

215 networks, and requires low operator expertise, costs, and response time, but also offers high

216 reproducibility, species identification accuracy, and usability. The ANN algorithm is optimized

217 for testing datasets with high levels of species richness, in this case 740 species $(11,198$

218 individuals) of fishes, plants and butterflies. 
219 The predictive ability of the ANNs was affected by the high phenotypic similarity between

220 species in the analysis, for example small fish species such as those from the family Characidos

221 (Annex 1, Fig. 8). The magnitude of this error comes from low phenotypic differences of some

222 species that vary only in minor details, like teeth or fin radii, which hinders classification.

223 However, the error obtained on the neural network model has been low in other taxonomic

224 families (Table 3). Overall performance of the system achieved high accuracy and precision,

225 with $91.65 \%$ true positive fish identifications, $92.87 \%$ plant identifications, and $93.25 \%$ butterfly

226 identifications. The evaluation of results appears simple at first glance: the comparison of success

227 rates appears sufficient, however upon closer examination, the success rates in tests on closed

228 data sets strongly depend on the number of species and the ratio of test to training image samples.

229 The data sets with a lower species number have higher success rates, possibly explained by

230 species with very distinct morphological characteristics.

231 Direct observation of an individual through a taxonomic key is, to this day, the most widely used

232 technique for species recognition and classification. This technique not only assumes prior

233 knowledge in the area of taxonomy by those who apply it, but also training and experience to

234 achieve acceptable classification results. Training and experience are absolutely necessary for the

235 classification specialist, who must acquire an ability to distinguish specific characteristics of the

236 species. Therefore, by comparing extracted features of individual images with classifications of a

237 traditional taxonomist, would be that the algorithm proposed uses color as variations present in

238 each of the individuals that are acquired by the normalized central moments of entropy and

239 inertia. These measurements are carried out in the space of red, green, and blue (RGB).

240 Geometric information is captured by measuring the compactness, compatibility, the area, and

241 perimeter of an individual. The normalized central moments, when measured over the area of the

242 individual, also provide important information regarding the general description of the shape of 
243 the object (fish, butterfly, plant, etc.). All of these descriptors can be used to uniquely define a

244 function to a particular region of the individual. Roughness, which is important for differentiation

245 of individuals, is taken into account as part of the texture, which although less so, can also be

246 inferred by the normalized central moments. According to taxonomists and classification keys,

247 the individual characteristics to observe for performing classification are generally as follows:

248 morphological structures, color patterns, and sizes. These observations are taxonomical

249 characteristics of individuals that depend on the particular appreciation of the taxonomist. Thus,

250 taxonomists may bias the value of any given characteristic, and may also require relatively more

251 time than others to carry out the classification. Therefore, human subjectivity and time constraints

252 may be eliminated through the use of machine based classification.

\section{CONCLUSIONS}

254 The method we propose for feature extraction is totally alien to the human researcher, but it does

255 not depend on variations in how the researcher observes individual specimens of each species,

256 and therefore eliminates human subjectivity. For this reason, the method can be a rapid and

257 effective species identification tool. However, a human taxonomist is still required to train the

258 neural network defining species, and subjectivity or uncertainty is possible in this step. For this

259 reason, a good taxonomist is required when training the neural network to achieve successful

260 species classification.

261 The strength of this research is in its applicability to combat the "taxonomic crisis". In the past

262 three decades, many promising techniques for fish identification have emerged. Many of them are

263 based on genetics, interactive computer software, image recognition, hydro-acoustics, and

264 morphometric (Fischer, 2013). In our study, neural networks were tested as a possible method for

265 species identification. However, taking advantage of the fast performance of the ANNs and the

266 speed of modern PCs, further research should explore the applications of the ANN methodology 
267 to automate biomass estimation and real-time species classifications. This could produce useful

268 tools for both scientific and commercial use. Fischer (2013) concludes that the image recognition

269 methods are useful but their transferability and resolution are poor because species differ between

270 geographic regions. This is a clear obstacle to future ANN development and network

271 identification success. Our advances in this field in relation to species identification should be

272 developed for specific geographic regions and translated into user-friendly applications. We

273 support the development of species identification methods that are globally interchangeable but

274 also tailored to regional biodiversity composition.

\section{ACKNOWLEDGEMENTS}

276 We thank to Dr Paulo A. Buckup (Museu Nacional Rio de Janeiro), Dr José Luís Olivan

277 Birindelli (Museu de Zoologia da Universidade de São Paulo, Sao Paulo-Brasil), Dr Rosseval

278 Leite (Instituto Nacional de Pesquisas da Amazônia, Manaus-Brasil), Dra Gema Solís (Museo

279 Nacional de Ciencias Naturales, Madrid-España), Dr Efrain Rubio Rincon (Colección de

280 Referencia Biología Marina, Universidad del Valle, Cali-Colombia), and Dra Andrea Polanco

281 (Instituto de Investigaciones Marinas y Costeras, Santa Marta- Colombia) to allow to photograph

282 the fish lots, Cesar Uribe for his helping on data analysis, Jonathan Bustamante Alvarez through

283 the photos on figure 1 (Universidad de Antioquia). Thanks are extended to Benjamin Branoff,

284 Aaron Hogan, and Paul Furumo for reviewing the English.

\section{REFERENCES}

286 Dayrat B. 2005. Towards integrative taxonomy. Biological Journal of the Linnean Society 85:

$287 \quad 407-415$.

288 Erickson G, Jörgensen P, Jörgensen C, Riccardi G, Ronquist F, van Engelen R. 2007.

289 Morphbank: Web Image Database Technology for Comparative Morphology and

290 Biodiversity Research http://www.morphbank.net/ (accessed on 18 October 2013) 
291 Fischer J. 2013. Fish identification tools for biodiversity and fisheries assessments: review and guidance for decision-makers. FAO, Rome ed: FAO Fisheries and Aquaculture 107.

293 Flavia. (2009). Flavia at a glance. http://flavia.sourceforge.net/ (accessed on 28 October 2013)

294 Flusser J, Suk T. 1993. Pattern-recognition by affine moment invariants. Pattern Recognition 295 26: 167-174.

296 Gaston KJ, O'Neill MA. 2004. Automated species identification: why not? Royal Society Philosophical Transactions Biological Sciences 359: 655-667.

298 Glasbey C. 1996. Handbook of pattern recognition and computer vision - Chen, CH, Pau,LF, 299 Wang,PSP. Journal of Classification 13: 350-352.

300 Grosjean P, Picheral M, Warembourg C, Gorsky G. 2004. Enumeration, measurement, and 301

Marqués de Sá J. 2001. Pattern Recognition: Concepts, Methods and Applications. Springer: Springer.

Hu M-K. 1962. Visual pattern recognition by moment invariants: Information Theory, IRE Transactions. 179 - 187.

Kang S-H, Song S-H, Lee S-H. 2012. Identification of butterfly species with a single neural network system. Journal of Asia-Pacific Entomology 15: 431-435.

Lancieri L, Boubchir L. 2007. Using multiple uncertain examples and adaptative fuzzy reasoning to optimize image characterization. Knowledge-Based Systems 20: 266-276.

Mayo M, Watson AT. 2007. Automatic species identification of live moths. Knowledge-Based Systems 20: 195-202. 
316 Novotny P, Suk T. 2013. Leaf recognition of woody species in Central Europe. Biosystems

317 Engineering 115: 444-452.

318 O'Neill MA. 2007. DAISY: a practical computed-based tool for semi-automated species

319 identification. Systematics Association Special Volume Series 74: 101-114.

320 Parker DB. 1987. Optimal Algorithms for Adaptive Networks: Second order Back Propagation, 321 Second Order Direct Propagation, and Second Order Hebbian Learning: Proceedings of 322 the IEEE First International Conference on Neural Networks \rm San Diego, CA. 593323600.

324 Rosenblatt. F. 1958 The Perceptron: A Probabilistic Model for Information Storage and 325 Organization in the Brain. Psychological Review 65: 386-408

326 Rother C, Kolmogorov V, Blake A. 2004. "GrabCut" - Interactive foreground extraction using 327 iterated graph cuts. Acm Transactions on Graphics 23: 309-314.

328 Rumelhart DE, Hinton GE, Williams RJ. 1986. Learning representations by back-propagating 329 errors. Nature 323: 533-536.

330 Smith BP, Brier ME. 1996. Statistical approach to neural network model building for gentamicin peak predictions. Journal of Pharmaceutical Sciences 85: 65-69.

332 Storbeck F, Daan B. 2001. Fish species recognition using computer vision and a neural network. $333 \quad$ Fisheries Research 51: 11-15.

334 Strachan NJC, Nesvadba P, Allen AR. 1990. Fish species recognition by shape-analysis of 335 images. Pattern Recognition 23: 539-544.

336 Werbos PJ. 1974. Beyond Regression: New Tools for Prediction and Analysis in the Behavioral 337 Sciences: Harvard University.

338 White DJ, Svellingen C, Strachan NJC. 2006. Automated measurement of species and length 339 of fish by computer vision. Fisheries Research 80: 203-210. 
340 Wu CH. 1997. Artificial neural networks for molecular sequence analysis. Computers \&

$341 \quad$ Chemistry 21: 237-256.

342 Zhang GQ, Patuwo BE, Hu MY. 1998. Forecasting with artificial neural networks: The state of 343 the art. International Journal of Forecasting 14: 35-62.

344 Zion B, Alchanatis V, Ostrovsky V, Barki A, Karplus I. 2007. Real-time underwater sorting of 345 edible fish species. Computers and Electronics in Agriculture 56: 34-45. 


\section{Table $\mathbf{1}$ (on next page)}

Table 1

Features extracted 
Table 1. Features extracted

\begin{tabular}{lll}
\hline Type & Variable & Description \\
\hline Geometrical & $A$ & Area \\
& $P$ & Perimeter \\
& $D$ & Diameter \\
& $C$ & Compatibility \\
& $C o$ & Compactness \\
Texture & $S$ & Solidity \\
& $u$ & Median \\
& $\delta^{2}$ & \\
& $E_{r, \boldsymbol{\theta}}$ & \\
& $H_{r, \boldsymbol{\theta}}$ & \\
& $H G_{r, \boldsymbol{\theta}}$ & Uniformity \\
& $I_{i}$ & Entropy co-occurrence \\
& $\varphi_{1}$ & Homogeneity \\
& $I_{1}, I_{2}$ & Inertia \\
Morphologica & & Hu1 \\
& & Amil-Ami2 \\
\hline
\end{tabular}


Table 2 (on next page)

Parameters used in ANN

FC (Fish collection); parameters used in neural network systems. 
Table 2. FC (Fish collection); parameters used in neural network systems.

\begin{tabular}{|l|l|l|l|l|l|}
\hline Data set & Learning & $\begin{array}{l}\text { Number of } \\
\text { generations }\end{array}$ & $\begin{array}{l}\text { Number of } \\
\text { Hidden } \\
\text { layers }\end{array}$ & $\begin{array}{l}\text { Number of } \\
\text { input layers }\end{array}$ & $\begin{array}{l}\text { Number of } \\
\text { output layers (\# } \\
\text { species) }\end{array}$ \\
\hline FC-MZUSP & 0.2 & 95000 & 200 & 15 & 100 \\
\hline FC-INPA & 0.15 & 100000 & 180 & 15 & 91 \\
\hline FC-MNRJ & 0.25 & 78000 & 60 & 15 & 14 \\
\hline FC-INVEMAR & 0.3 & 84000 & 250 & 15 & 189 \\
\hline FC-CIUA & 0.12 & 90000 & 60 & 15 & 33 \\
\hline FC-CRBMUV & 0.35 & 140000 & 300 & 15 & 172 \\
\hline FC-MNCN & 0.2 & 110000 & 250 & 15 & 98 \\
\hline FLAVIA & 0.1 & 50000 & 60 & 15 & 32 \\
\hline BUTTERFLIES & 0.5 & 50000 & 35 & 15 & 11 \\
\hline
\end{tabular}


Table 3(on next page)

Results of ANN

FC (Fish collection); results of ANN tests with species for 15 features 
Table 3. FC (Fish collection); results of ANN tests with species tests for 15 features

\begin{tabular}{|l|l|l|l|l|l|l|}
\hline \multicolumn{4}{|c|}{ Average Percentage of images (Training / test) } \\
\hline Data set & Species & Images & $\mathbf{6 0 / 4 0}$ & $\mathbf{7 0 / 3 0}$ & $\mathbf{8 0 / 2 0}$ & $\mathbf{9 0 / 1 0}$ \\
\hline FC-MZUSP & 100 & 1718 & 76.67 & 81.34 & 83.34 & 88.31 \\
\hline FC-INPA & 91 & 1640 & 76.29 & 78.94 & 84.44 & 89.93 \\
\hline FC-MNRJ & 14 & 422 & 82.62 & 87.18 & 90.56 & 91.65 \\
\hline FC-INVEMAR & 189 & 1703 & 76.72 & 84.03 & 86.45 & 88.08 \\
\hline FC-CIUA & 33 & 472 & 83.08 & 86.99 & 90.19 & 91.77 \\
\hline FC-CRBMUV & 172 & 2392 & 77.36 & 85.21 & 87.29 & 88.85 \\
\hline FC-MNCN & 98 & 959 & 72.34 & 86.21 & 88.15 & 89.11 \\
\hline FLAVIA & 32 & 1800 & 68.79 & 88.48 & 91.61 & 92.87 \\
\hline BUTTERFLIES & 11 & 92 & 73.62 & 80.43 & 88.83 & 93.25 \\
\hline
\end{tabular}




\section{Figure 1}

Samples of some species

Samples of some species data set : 1)Curimata mivartii 2)Leporinus striatus3)Ctecolucius hujeta4)Cinopotamus magdalenae5)Astyanax magdalenae6)Roeboides occidentalis7)Genycharax tarpon8)Cyphocharax magdalenae9)Hemibrycon decurrens 10)Brycon medemi11)Lebiasina multimaculata12)Hemibrycon dentatus13)Triporheus magdalenae14)Characidium phoxocephalum15)Leporinus muyscorum16)Hemibrycon boquiae17)Brycon hennir18Characidium caucanum19)Roeboides dayi20)Astyanax fasciatus21)Argopleura magdalenensis22)Apteronotus eschemeyeri23)Eigenmannia virescens.

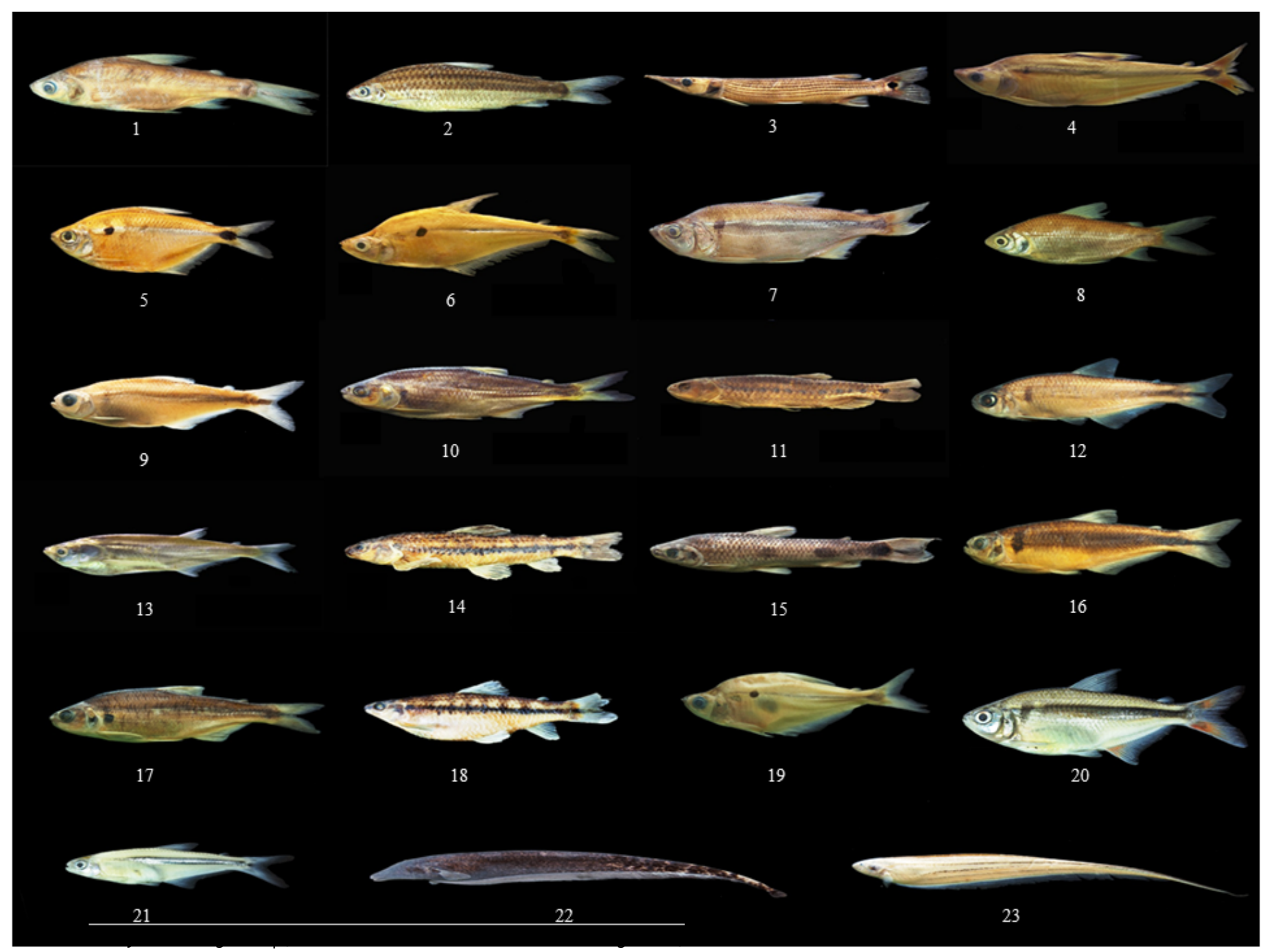




\section{Figure 2}

Samples of plants

Samples of our data set: 1)Phyllostachys edulis2)Aesculus chinensis3)Berberis anhweiensis4)(ercis chinensis5)Indigofera tinctoria6)Acer Dalmatum7)Phoebe zhennan8)Kalopanax septemlobus9)Cinnamomum japonicum10)Koelreuteria paniculata11)Ilex macrocarpa12)Pittosporum tobira13)Chimonanthus praecox14)Cinnamomum camphora15)Viburnum awabuki16)Osmanthus fragrans17)Cedrus deodara18)Ginkgo biloba19)Lagerstroemia indica20)Nerium oleander21)Podocarpus macrophyllus22)Prunus yedoensis23)Ligustrum lucidum24)Tonna sinensis25)Prunus persica26)Manglietia fordiana27)Acer buergerianum28)Mahonia bealei29)Magnolia grandiflora30)Populus Canadensis31)Liriodendron chinense32)Citrus reticulate. 


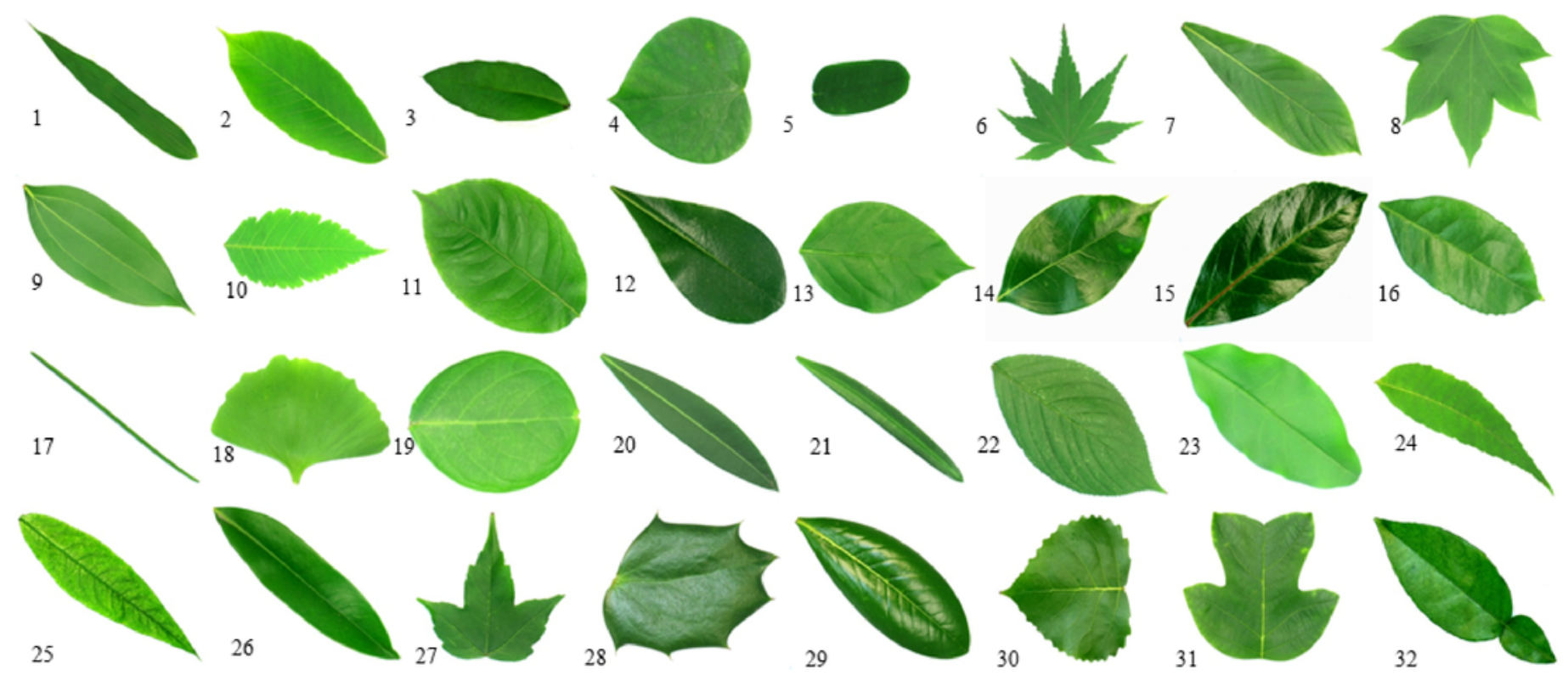




\section{Figure 3}

Samples of butterflies

Samples of our data set: 1)Agraulis vanillae2)Anthocharis midea3)Ascia monuste4)Danaus gilippus5)Danaus plexippus6)Dryas iulia7)Enodia portlandia8)Glutophrissa Drusilla9)Heliconius charithonia10)Pieres rapae11)Pontia protodice.

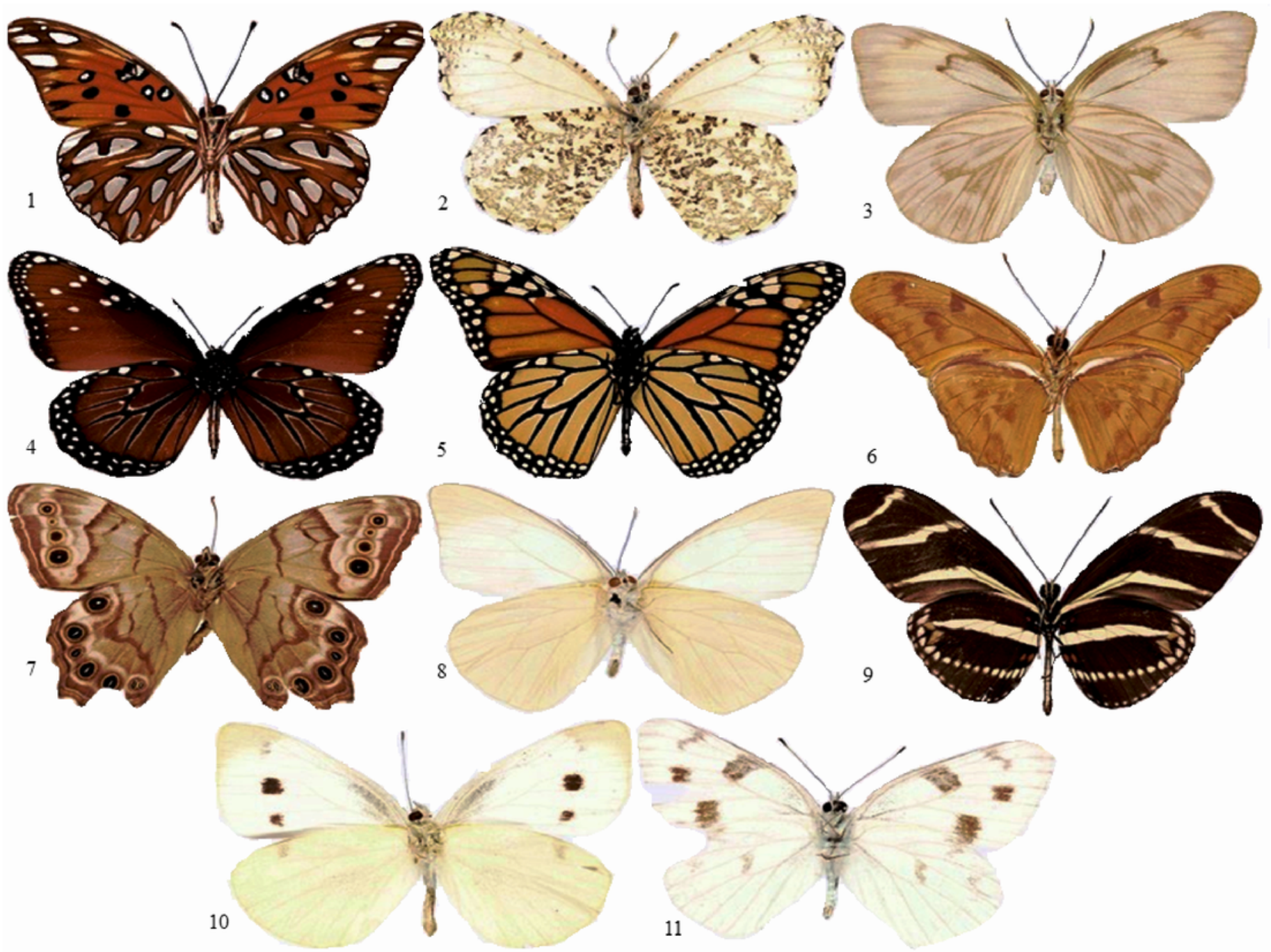


Figure 4

\section{Architecture}

System architecture

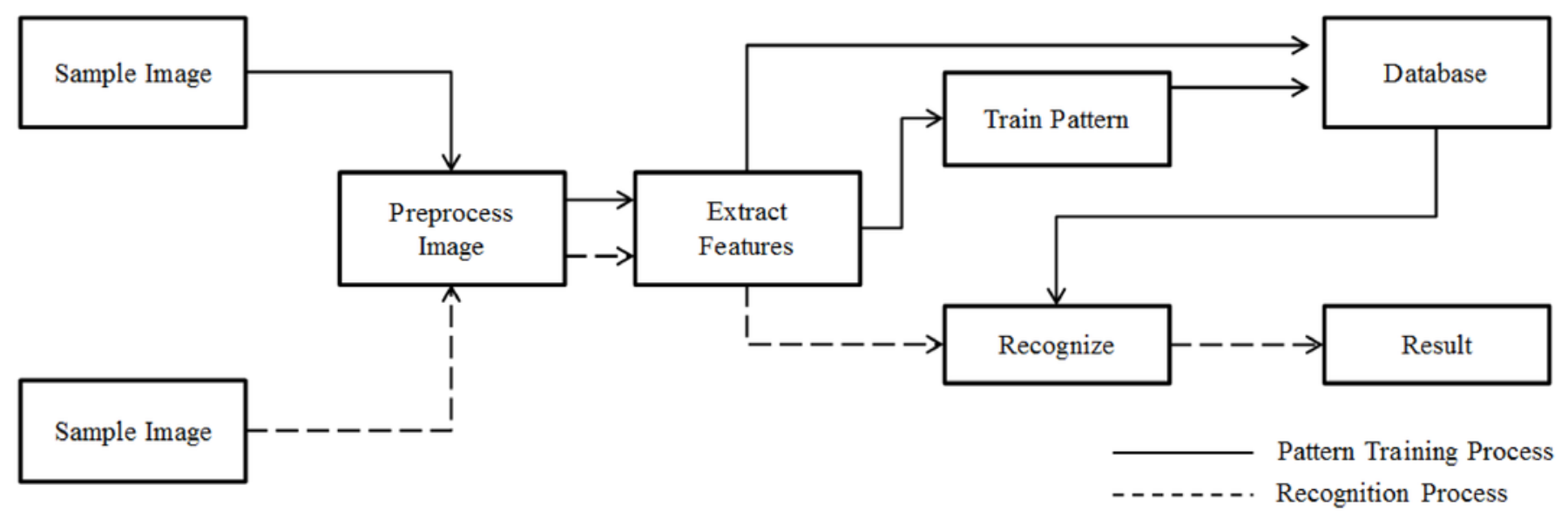




\section{Figure 5}

Image processing

Image processing 1) jpg image, 2) Image background is removed, 3) grayscale image, 4) smoothing filter, 5) median filter, 6) binarized image, 7) contour image 8) skeletonized image.

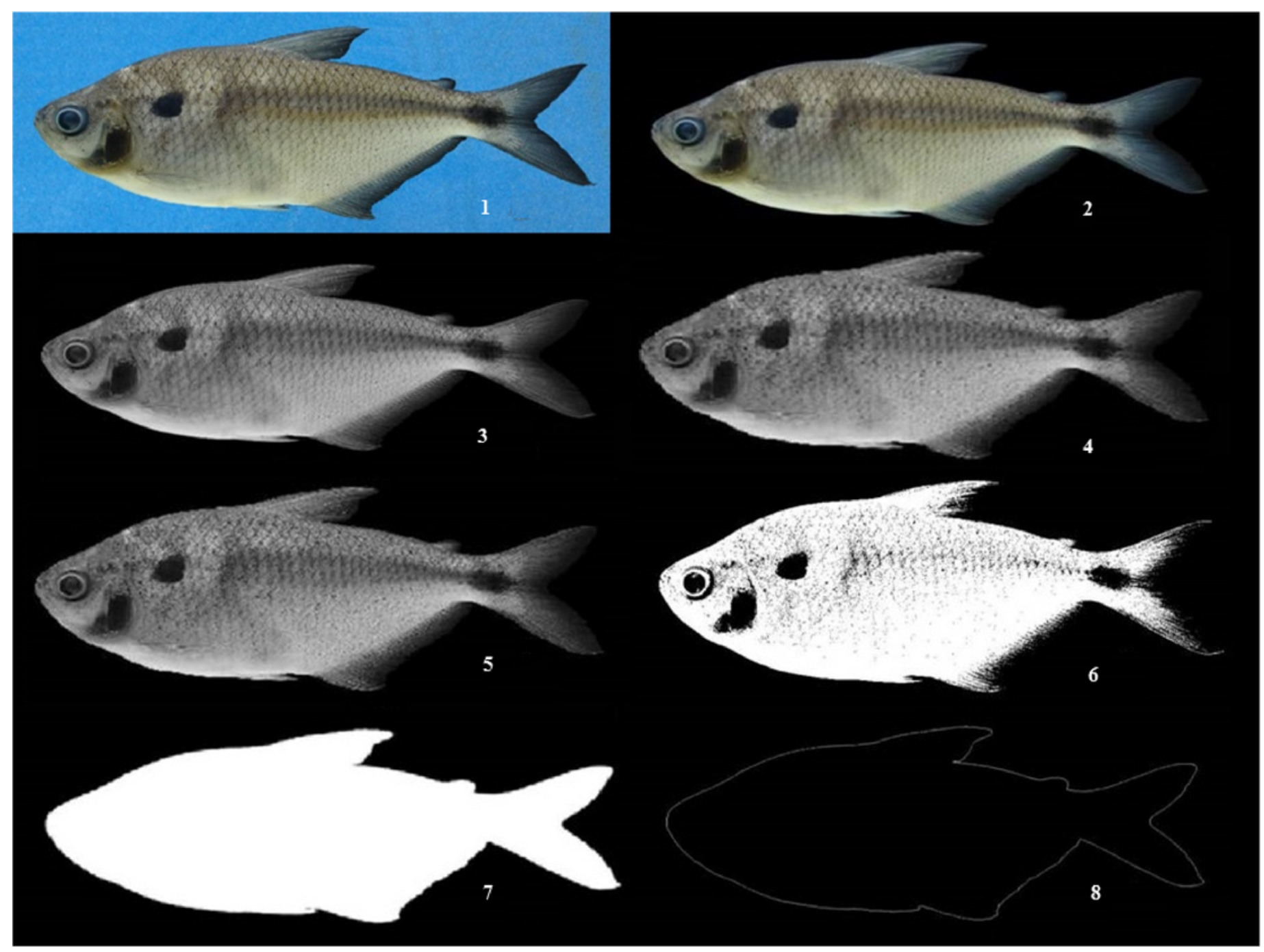


Figure 6

Multilayer perceptron

General architecture of a multilayer perceptron. $<!--$

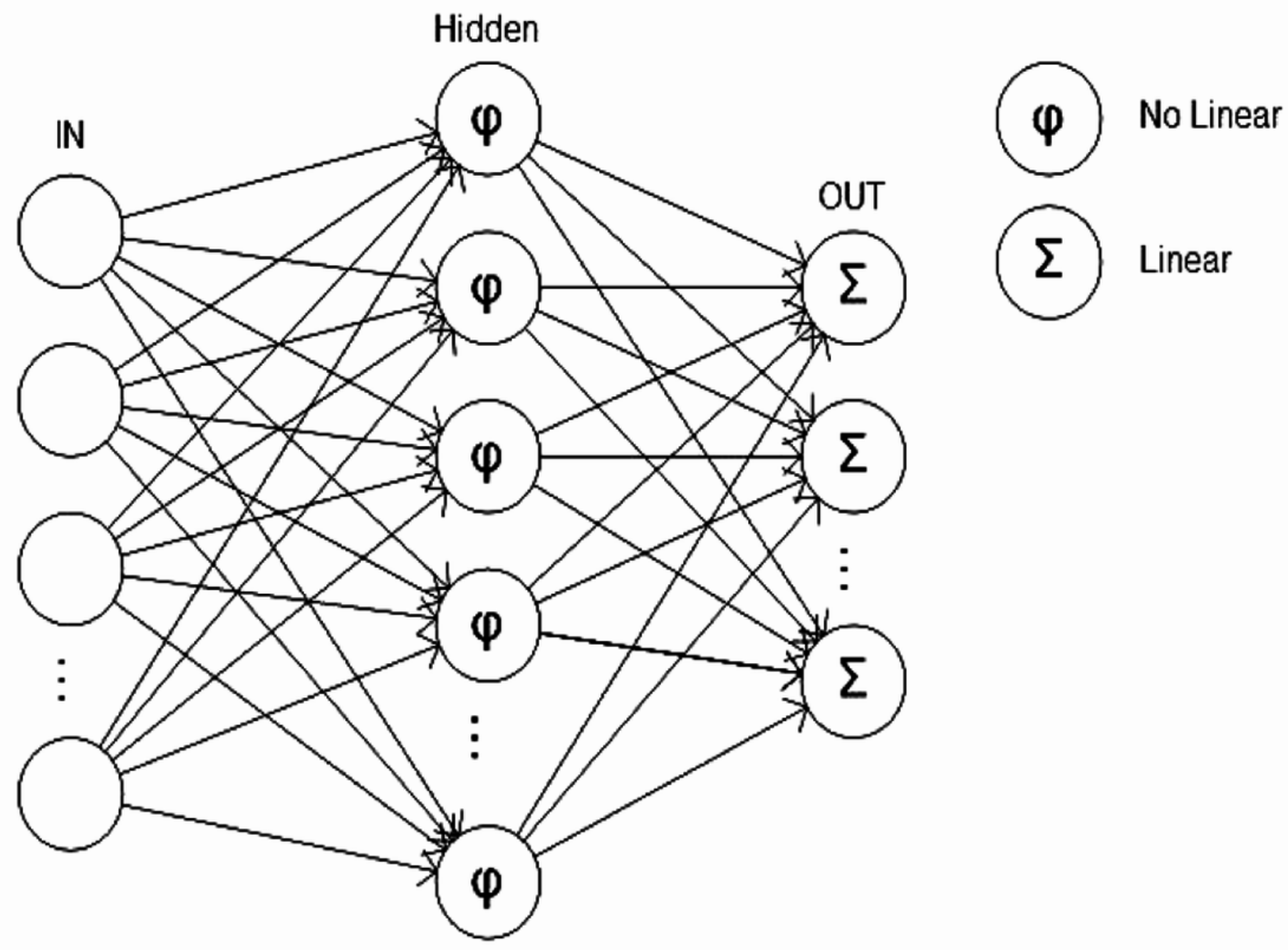


Figure 7

Rate and the number of neurons

Relationship between the success rate and the number of neurons for each neural network.
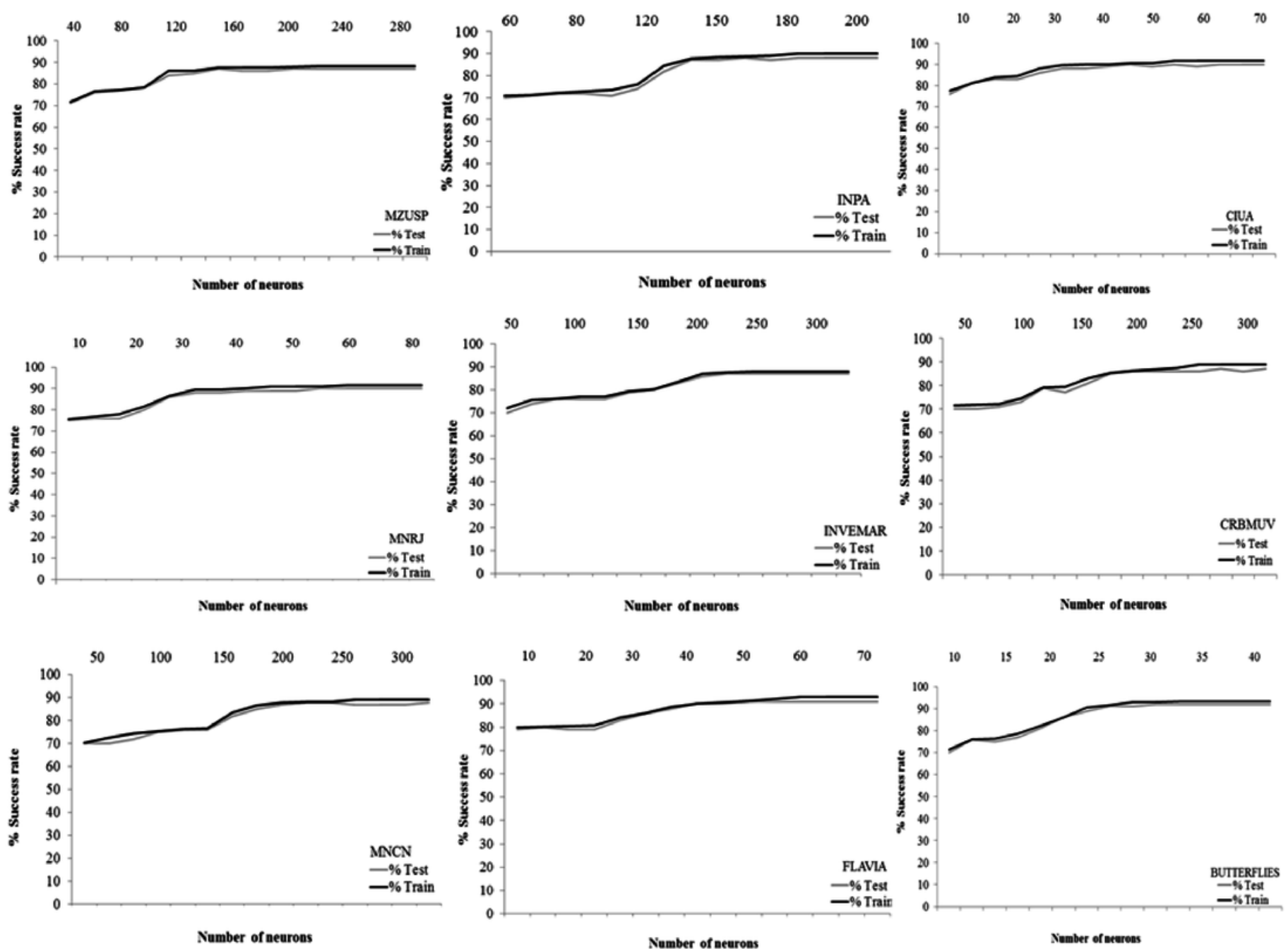


\section{Figure 8}

Figure 8

An example of species confusion in the genus Astyanax 1)Astyanax magdalenae, 2)Astyanax caucanus, 3)Astyanax fasciatus, and4) Astyanax microlepis.

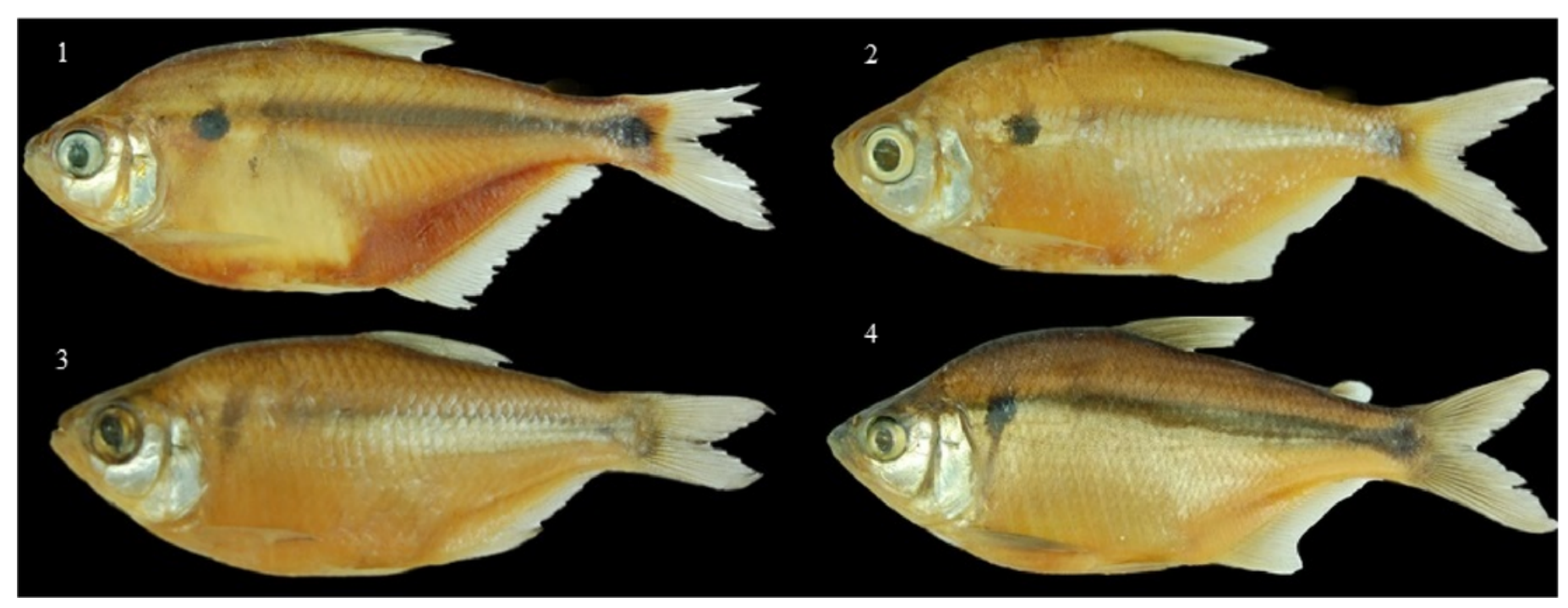

